\title{
Prenatal diagnosis of fetal chromosomal abnormalities: Report of an 18-year experience in a Brazilian public hospital
}

\author{
Rejane G. Kessler, Maria Teresa V. Sanseverino, Sandra Leistner-Segal, José A.A. Magalhães \\ and Roberto Giugliani \\ Serviço de Genética Medica, Hospital de Clínicas de Porto Alegre, Porto Alegre, RS, Brazil.
}

\begin{abstract}
The study of the fetal karyotype became an important tool for the fetal diagnosis of genetic diseases in the 1970s. Although application of this test has remained very restricted in Brazil, we had 905 referrals for prenatal fetal karyotyping between 1989 and 2007. In 879 cases, a fetal karyotype was obtained. We detected 74 abnormal karyotypes $(8.4 \%)$, the majority being found when the prior indication was fetal malformation. When obtaining amniotic fluid or chorionic villus samples was difficult, alternative fetal materials (urine, cystic hygroma, cystic lung, intreperitoneal and cerebrospinal fluids) were collected and we had success in obtaining karyotypes in all 13 cases. Although, the option of terminating abnormal pregnancies does not legally exist in Brazil, the information gained in assessing the prognosis of on-going pregnancies or estimating recurrence risks justifies prenatal diagnosis of chromosome abnormalities. We conclude that, in keeping with the policy in most other countries, prenatal cytogenetic analysis is strongly recommended in high-risk pregnancies for fetal abnormalities. However, the unique aspect of this type of study is not its rarity in world terms, but its rarity in Brazil. This argues that Brazilian health policy on prenatal diagnosis requires reforming to make it much more widely available within the public health care sector.
\end{abstract}

Key words: prenatal diagnosis, chromosomal abnormalities, fetal malformations.

Received: January 31, 2008; Accepted: August 25, 2008.

\section{Introduction}

During the last decades the study of fetal karyotypes has become a very important tool for genetic counseling on recurrence risk and/or fetal chromosome diagnosis of atrisk pregnancies (Magalhães, 2001). Invasive prenatal diagnosis continues to be the standard method for searching for chromosomal aneuploidies or other genetic diseases (Bui, 2007). Prenatal diagnosis of cytogenetic abnormalities is now widely recognized as a reliable method with an acceptable risk for couples at high risk of giving birth to a child with clinically significant chromosome abnormalities (Caron et al., 1999). Despite the fact that in Brazil amniocentesis and CVS were first introduced by Nazareth et al. (1981) and Gollop et al. (1988) respectively, there is still no public health care policy for application of cytogenetic prenatal diagnosis. As in other developing countries, this test is mostly confined to expensive private clinics, which means that it is rarely available for the great majority of pregnant women who depend on public medical services.

Send correspondence to Rejane Gus Kessler. Serviço de Genética Medica, Hospital de Clinicas de Porto Alegre, Rua Ramiro Barcelos 2350, 90035-903 Porto Alegre, RS, Brazil. E-mail: rkessler@ hcpa.ufrgs.br.
Nevertheless, we have been offering this test in our public hospital since 1989. Prenatal diagnosis is a very restricted test in Brazil, mainly because induced abortion, even indicated by fetal genetic disease, is not legally allowed. Despite this, we have had 905 referrals for fetal karyotyping since it was first offered by our clinic in 1989. In the first four years, we had an average of 80 cases/year and this number decreased in the following ten years to 45 cases/year. In the last four years this number decreased even further, to 35 cases/year. This will be discussed later.

Even with the development of modern techniques, cell culture failure remains one of the main obstacles to be overcome. In order to improve the chance of getting a karyotype result, alternative fetal samples, such as urine or cystic hygroma fluid were used for chromosome analysis when malformations were found in the fetus and availability of conventional tissues was limited. The purposes of this study were: 1) to describe the most frequent indications for karyotyping the fetus in our socio-economic conditions; 2) to estimate the frequency of the most common prenatal chromosome abnormalities in patients from the Hospital de Clinicas de Porto Alegre; 3) to assess the cytogenetic results obtained with alternative tissue samples compared to amniocytes and chorionic villi. 


\section{Materials and Methods}

Cytogenetic findings were retrospectively reviewed from 1989 to 2007 in 905 pregnant women, with a mean maternal age of 32.7 years, and mean gestational age of 22.7 weeks. Those women underwent prenatal cytogenetic evaluation only after a genetic counseling session, which means that risks, methods and indications were explained to the family. All samples were collected by a single gynecologist. The method used for sample collection was transabdominal punction guided by ultrasound. Samples were obtained for all patients, even in cases of lack of amniotic fluid, when alternative fluids were collected. Amniotic fluid, or any other fetal sample collected, were cultivated in long-term cell cultures, with Amniomax medium, at $37^{\circ} \mathrm{C}$ in $\mathrm{CO}_{2}$ incubator. Cordocentesis followed the standard blood culture that means, short-term culture $(72 \mathrm{~h})$ at $37^{\circ} \mathrm{C}$, and no requirement for a $\mathrm{CO}_{2}$ incubator. We used standard Giemsa-banding staining technique for all chromosome analyses.

\section{Results}

The most frequent indications for prenatal cytogenetic diagnosis were advanced maternal age (with an average of 39.9 years old and mean gestational age of 18.7 weeks), abnormal findings on fetal ultrasound, a previous child with chromosomal abnormalities, and increased nuchal translucency (Table 1). Despite advanced maternal age being the most frequent indication for prenatal diagnosis, the majority of aberrant karyotypes were found when the indication was a fetal malformation detected by ultrasound. On the other hand, although the history of a previous child with Down syndrome was a relatively frequent indication, we did not find any positive cases in this group.

From the 905 prenatal cytogenetic analysis performed, we failed to obtain results in $26(2.8 \%)$. Among the 879 karyotypes obtained, 74 (8.4\%) were abnormal. (Table 1). Numerical abnormalities were found in 64 cases $(7.3 \%)$, and structural aberrations in 10 cases $(1.1 \%)$. The majority of numerical chromosomal abnormalities were autosomal trisomies. Trisomy 21 was the most frequent $(28 ; 3.2 \%)$, and the second most frequent was trisomy 18 $(24 ; 2.7 \%)$. Interestingly, trisomy 18 was almost entirely restricted to the group of "fetal abnormalities detected by ultrasound" and none was detected in the "increased nuchal translucency" group $(\mathrm{p}<0.001)$. On the other hand, the difference in the frequencies of trisomy 21 between these two types of ultrasound prescreening was not statistically significant $(\mathrm{p}=0.096)$. Trisomy 13 was found in six cases $(0.7 \%)$, monosomy $\mathrm{X}$ in one $(0.6 \%)$ and one case showed triploidy. Among structural chromosomal aberrations, translocations were the most frequent, and were detected in four out of the 879 cases analyzed $(0.45 \%)$ : reciprocal translocations in two cases and Robertsonian translocations in two others. Marker chromosomes were found in three cases, deletions in two cases and an inversion was present in one case.

In 13 cases alternative fluid samples were obtained (Table 2). The reasons for collecting alternative materials were lack of amniotic fluid in seven cases of kidney pathol-

Table 1 - Indications for invasive prenatal diagnosis and abnormal karyotypes.

\begin{tabular}{|c|c|c|c|c|}
\hline Primary indication & Total number of cases $(\%)$ & Karyotypes obtained & Abnormal karyotypes (\%) & Type of abnormalities (n) \\
\hline Advanced maternal age & $235(25.9)$ & 227 & $13(5.7)$ & $\begin{array}{l}\text { Trisomy } 21 \text { (10) } \\
\text { Trisomy } 18 \text { (3) }\end{array}$ \\
\hline $\begin{array}{l}\text { Fetal malformation at ultrasound } \\
\text { other than increased nuchal } \\
\text { translucency }\end{array}$ & $177(19.5)$ & 169 & $38(22.5)$ & $\begin{array}{l}\text { Trisomy } 18(19) \\
\text { Trisomy } 21(9) \\
\text { Trisomy } 13(4) \\
\text { 47,, , }+ \text { mar }(2) \\
45, X(1) \\
\text { Triploidy (1) } \\
\text { 46,XX+13,der(13;14)(q10;q10) (1) } \\
46, X Y, \operatorname{del}(18)(\mathrm{p} ?)(1)\end{array}$ \\
\hline Previous child with trisomy & $125(13.8)$ & 123 & 0 & 0 \\
\hline Increased nuchal translucency & $65(7.1)$ & 63 & $9(14.3)$ & $\begin{array}{l}\text { Trisomy } 21(8) \\
47, X Y,+\operatorname{mar}(1)\end{array}$ \\
\hline Non immune Fetal hydrops & $54(5.9)$ & 50 & $10(20)$ & $\begin{array}{l}45, \mathrm{X}(4) \\
\text { Trisomy } 13(2) \\
\text { Trisomy } 18(2) \\
\text { Trisomy } 21(1) \\
46, \mathrm{XY},+14, \operatorname{der}(14 ; 21)(\mathrm{q} 10 ; \mathrm{q} 10)(1)\end{array}$ \\
\hline Others & $249(27.5)$ & 247 & $4(1.6)$ & $\begin{array}{l}\text { 46,XX,+der(18)add(18)(p11)(1) } \\
46, \mathrm{XX}, \mathrm{t}(15 ; 16)(\mathrm{q} 21 ; \mathrm{p} 12)(1) \\
46, \mathrm{XX}, \operatorname{inv}(12)(\mathrm{q} 13 \mathrm{q} 23)(1) \\
46, \mathrm{XY}, \mathrm{t}(7 ; 10)(\mathrm{p} 21 ; \mathrm{q} 21)(1)\end{array}$ \\
\hline Total & 905 & 879 & $74(8.6 \%)$ & \\
\hline
\end{tabular}


Table 2 - Source of fetal material for karyotyping and success rate of cell cultures.

\begin{tabular}{lcccc}
\hline Fetus sample & Number of cases (n) & Culture success (n) & Success rate (\%) & Gestational age in weeks (average) \\
\hline Amniotic fluid & 777 & 755 & 97.1 & 28.8 \\
CVS & 61 & 57 & 93.4 & 12.9 \\
Cord blood & 54 & 54 & 100 & 26.4 \\
Alternative fluids $^{\mathrm{a}}$ & 13 & 13 & 100 & 31.2 \\
\hline Total & 905 & 879 & 97.2 & 28.4 \\
\hline
\end{tabular}

${ }^{a}$ Bladder (6), cystic hygroma (2), intraperitoneal (2), displastic kidney (1), cystic lung (1), cerebrospinal (1) fluids.

ogies, therapeutic drainage to facilitate delivery in six cases due to ascitis $(n=2)$, abdominal cyst $(n=2)$, pulmonary cyst $(n=1)$ and hydrocephaly $(n=1)$. The gestational age varied from $18^{\text {th }}$ to $36^{\text {th }}$ weeks with a mean age of 27.3 weeks. We had success in culturing these materials and in obtaining karyotypes in all cases (Table 2).

\section{Discussion}

Prenatal diagnosis has become a major aid to genetic counseling and for this, several important areas of technology have evolved, especially cytogenetic prenatal diagnosis, using analysis of cultured cells from the amniotic fluid at mid-trimester. Because of its high reliability and safety record with the lowest fetal loss and embryonic damage, amniocentesis has become the most common practice for prenatal diagnosis (Park et al., 2001). However, CVS (chorionic villus sample) has gained popularity as a successful first trimester prenatal diagnostic technique since the mid 1980s (Brambati et al., 1998), probably because of the advantage of establishing a diagnosis some weeks earlier in the pregnancy. Cordocentesis is a procedure used to obtain a sample from fetal blood directly from the umbilical cord in cases where amniocentesis is not possible or is used to give a quick result only in high-risk cases since procedure related pregnancy loss is high (Costa et al., 1998).

Prenatal cytogenetic diagnosis using the above techniques was established in many countries, including Brazil (Gollop et al., 1993; Pinto Jr, 2002), and has been performed for more than 18 years at the Hospital de Clínicas de Porto Alegre. During this period, the number of cytogenetic analyses has decreased by almost 50\% per year in the Hospital and this can be explained by two facts: the introduction of nuchal translucency (NT) as a reliable screening method, and in the last four years medical insurance has provided payment for this exam, making it more accessible for the population. We would question whether NT alone is reliable to detect all forms of cytogenetic abnormality, since no cases of trisomy 18 were found in our NT sample $(n=65)$. On the other hand, when other forms of fetal abnormality detected by ultrasound were considered, then a frequency of trisomy 18 emerged which was even higher than trisomy 21 within this group. Intriguingly, our results suggest that prior diagnosis of fetal malformations using ultrasound is particularly efficacious for detecting trisomy 21 with the nuchal translucency test and, for trisomy 18, when other types of malformation are detected. However, Cheng et al. (2003) detected five cases of trisomy 18 among 171 instances of increased NT. This discrepancy might be due to our small size sample. Anyway, our results indicate that although ultrasound for nuchal translucency is strongly advised, any ultrasound prescreening should not be restricted to nuchal translucency, but should include also more generalized types of malformation, such as heart abnormalities, which are claimed to be present in almost all trisomy $18 \mathrm{fe}$ tuses. However, we feel that NT measurement used as a routine screening has decreased the number of referrals due to advanced maternal age, which has a low specificity, and has increased relatively the number of referrals for fetal abnormalities with a higher specificity. However, it has to be realized that tests such as nuchal translucency are not replacements for cytogenetic analysis, but provide strong indications for performing cytogenetic analysis in abnormal cases. The same arguments apply to serum screening in pregnant women. In some countries, such as the United Kingdom, increased maternal age is no longer applied as the sole referral indication for chromosome prenatal diagnosis; it is the combination of maternal age, serum screening and nuchal translucency and detection of other abnormalities by ultrasound which determines the validity of performing subsequent expensive cytogenetic analysis. However, all this is predicated on having all methods supported under the public health care system.

In a preliminary genetic counseling session, the approaches, methods and correct indications, are discussed with the family. In our sample, the history of a previous child with Down syndrome is the third more frequent indication. Although the risk of a recurrent trisomy is well established (Warburton et al, 2004), the risk is low and, not surprisingly, we did not find any recurrent case. Considering the current economic limitations to offer prenatal tests in our country, we propose that higher priority for the indication of prenatal diagnosis should be given to pregnancies where a malformation is detected on ultrasound scan than for couples who had a previous Down child, unless Down syndrome was caused by a Robertsonian translocation carried by one of the parents. This latter also assumes that post natal cytogenetic screening of all Down patients and, where necessary, their parents has occurred 
already to identify those families with a high recurrence risk due to one of the parents being a carrier of a translocation involving chromosome 21. It is such families that will derive the most benefit from prenatal diagnosis. With such a directed policy we, and other centers, would be able to provide more opportunity for poor families with higher risks for fetal abnormalities to be assisted by prenatal diagnosis within the public health care system in Brazil.

The results of fetal cytogenetic abnormalities in our study are similar to those reported in the literature (Caron et al., 1999; Carothers et al., 1999; Quintana et al., 1999). Several studies have shown that Down syndrome is the most common and clinically significant cytogenetic abnormalities detected in prenatal cytogenetic studies (Mathews et al., 1992; Carothers et al., 1999), followed by Edwards Syndrome (Song et al., 1997; Han et al., 2000). This was also found to be the case in our own series. The frequency of chromosomal abnormalities in the general population is estimated to be $0.5 \%$ of live births, but the frequency within the high-risk population is higher (around $5 \%$, as observed in newborns with malformation by Nazer et al., 2003, in Chile). The frequency of chromosomal abnormalities in our sample was even higher (8.5\%) than other studies (Park et al., 2001), probably because our Medical Genetic Service, as a reference center, receives patients who have been screened already by physicians in other Centers (without Genetic Services available) and are, therefore, more prone to having a chromosomal abnormality due to ultrasound alterations or familial history.

Karyotyping unconventional fetal samples, when it is difficult to obtain the traditional ones, is not a very common approach in most laboratories (Donnenfeld et al., 2001; Gole et al., 1997). We used this alternative when necessary and achieved a $100 \%$ success rate on an admittedly limited sample of 13 cases; however, the success rate is higher than that observed in other studies (Teoh et al., 1996; Donnenfeld et al., 2001).

Although, the option of terminating genetically abnormal pregnancies does not legally exist in Brazil, the information gained in assessing the prognosis of on-going pregnancies or estimating recurrence risks for future family planning justifies prenatal diagnosis of chromosome abnormalities. In our sample the three most frequent indications were advanced maternal age, fetal malformation at ultrasound and a previous child with trisomy. However, the majority of aberrant karyotypes were found in the group with a fetal malformation detected by ultrasound and, as argued above, this opens up the possibility of triaging the initial referral group and being more efficient in deriving the maximum benefit to the maximum number of patients under limited resources.

Although, the benefit of using "alternative" fetal samples for karyotyping is marginal in terms of numbers this approach can provide a karyotype result to high-risk families in situations where it has proven impossible to derive traditional tissues for analysis, even in advanced gestational age.

In general the analysis of our data supports the contention that the wide practice performed in many other countries of prenatal cytogenetic analysis being made available to the whole population and performed routinely in high-risk pregnancies, should also take place in Brazil within the public health care sector and not be almost entirely confined to the private care sector, as at present. However, a solid public health care policy for prenatal diagnosis needs to be established in which the distribution of facilities and reasonable coverage of expenditures has to be evaluated.

\section{References}

Brambati B, Tului L, Cislaghi C and Alberti E (1998) First 10000 chorionic villus samplings performed on singleton pregnancies by a single operator. Prenat Diagn 18:255-266.

Bui TH (2007) Prenatal cytogenetic diagnosis: Gone FISHing, BAC soon. Ultrasound Obstet Gynecol 30:247-251.

Caron L, Tihy F and Dallaire L (1999) Frequencies of chromosomal abnormalities at amniocentesis: Over 20 years of cytogenetic analysis. Am J Med Genet 82:149-154.

Carothers AD, Boyd E, Lowther G, Ellis PM, Couzin DA, Faed MJW and Robb A (1999) Trends in prenatal diagnosis of Down syndrome and other autosomal trisomies in Scotland 1990 to 1994, with associated cytogenetic and epidemiological findings. Genet Epidemiol 16:179-190.

Cheng PJ, Liu CM, Chueh HY, Lin CM and Shoong YK (2003) First-trimester nuchal translucency measurement and echocardiography at 16 to 18 weeks of gestation in prenatal detection for trisomy 18. Prenat Diagn 23:248-251.

Costa D, Borrell A, Soler A, Carrio A, Margarita E, Ballesta F, Puerto B, Caballin MR and Fortuny A (1998) Cytogenetic studies in fetal blood. Fetal Diagn Ther 13:169-175.

Donnenfeld AE, Lockwood D and Lamb AN (2001) Prenatal diagnosis from cystic hygroma fluid: The value of fluorescence in situ hybridization. Am J Obstet Gynecol 185:1004-1008.

Gole LA, Anandakumar C, Bongso A, Chua TM, Wong YC and Ratnam SS (1997) Analysis of cystic hygroma, ascitic and pleural fluids by conventional lymphocyte culture and fluorescent in situ hybridization. Prenat Diagn 17:1151-1157.

Gollop TR, Eigier A, Naccache N, Bittencourt EA and Hauschild D (1988) Amostra de vilo corial por via transabdominal: Nota preliminar. Femina 16:767-768.

Gollop TR, Naccache NF, Campos IMA and Pieri PC (1993) Amostra de vilo corial: 1290 casos / Chorionic villus sampling: 1290 cases. Rev Bras Ginecol Obstet 15:84-87.

Han JR, Kim MY, Ahn HK, Cho JH, Ryu HM, Kim JM, Kim YM, Park SY, Han HK and Yang JH (2000) Comparison of the contribution rate of various prenatal screening methods for Down syndrome. Korean J Obstet Gynecol 43:1780-1785.

Magalhães JAA (2001) Medicina fetal. In: Freitas F, Martins Costa SH, Ramos JGL and Magalhães JAA (Eds) Rotinas em Obstetrícia. 4th edition. ArtMed, Porto Alegre, pp 38-47.

Mathews T, Navsaria D and Verma RS (1992) Prenatal diagnosis of 1,400 consecutive amniocentesis. Gynecol Obstet Invest 34:122-123. 
Nazareth HRS, Pinto Jr W and Andrade JAD (1981) Diagnóstico pré-natal de aberrações cromossômicas. Primeira experiência brasileira. Rev Bras Genet 3:459-470. (Abstract in English).

Nazer J, Antolini M, Juárez ME, Cifuentes L, Hubner ME, Pardo A and Castillo S (2003) Prevalence of chromosomal aberrations at birth in the Clinical Hospital of Universidad de Chile, 1990-2001. Rev Med Chil 131:651-8.

Park SY, Kim JW, Kim YM, Lee MH, Han JY, Kim YM, Yang JH and Ryu HM (2001) Frequencies of fetal chromosomal abnormalities at prenatal diagnosis: 10 years experiences in a single institution. J Korean Med Sci 16:290-293.

Pinto Jr W (2002) Diagnóstico pré-natal. Ciênc Saúde Coletiva 7:139-157. (Abstract in English).

Quintana JA, Quiñones OM, Méndez LA, Lavista MG, González CE and Hernández GP (1999) Resultados del diagnóstico prenatal cromosómico en Ciudad Habana. Rev Cuba Obstet Ginecol 25:153-158.

Song HK, Ryu HM, Kim MY, Kim ES, Yoo SJ, Lee YH, Choi SK and Han HW (1997) Prenatal diagnosis of down syndrome. Korean J Obstet Gynecol 40:2826-2832.

Teoh TG, Ryan G, Johnson J and Winsor EJ (1996) The role of fetal karyotyping from unconventional sources. Am J Obstet Gynecol 175:873-877.

Warburton D, Dallaire L, Thangavelu M, Ross L, Levin B and Kline J (2004) Trisomy recurrence: A reconsideration based on North American data. Am J Hum Genet 75:376-385.

Associate Editor: Peter L. Pearson

License information: This is an open-access article distributed under the terms of the Creative Commons Attribution License, which permits unrestricted use, distribution, and reproduction in any medium, provided the original work is properly cited. 